\title{
AN INVESTIGATION OF THE CENTRES OF PRESSURE UNDER THE FOOT WHILE WALKING
}

\author{
Michael Grundy, Blackburn, P. A. Tosh, R. D. Mcleish and L. Smidt, Manchester, England
}

From the University of Manchester Institute of Science and Technology, Department of Mechanical Engineering

The forces under the foot while walking have been measured using a high sensitivity force-plate of the strip-suspended type combined with simultaneous filming of the sole of the foot. The recording of data and the calculation and plotting of results were much simplified by computer aid. Normal and abnormal feet, both barefoot and shod, were investigated in sixteen subjects. It was found that in normal barefoot walking the forefoot carried a total load of the order of three times that of the heel. When footwear was worn the function of the forefoot was progressively reduced as the rigidity of the sole of the shoe increased. Painful conditions of the forefoot also produced a large reduction in the proportion of the total load transferred.

The forces which act on the sole of the foot in walking are of considerable interest to orthopaedic surgeons. Variations in their site and magnitude may reflect painful conditions of the foot or abnormalities in structure and function. The function of the foot may also be altered by footwear or appliances in ways which cannot be fully assessed without measurement of the forces involved. Early attempts to measure the forces were made by a variety of ingenious methods, reviewed by Elftman in 1934. These ranged from recording the impressions made by steel shot in a lead plate on which the subject stood, to a harp-like machine in which the tension produced by standing on the strings was determined by plucking them.

More dynamic tests were carried out by Elftman, who filmed the underside of a corrugated rubber mat which was viewed from beneath a glass plate. When the mat was walked upon the deformation of the corrugations was proportional to the load. Similarly, Barnett (1954) devised a box of vertical Perspex rods whose vertical displacement into a rubber support could be filmed from the side when a subject walked on their upper surface. These methods have the disadvantage that the deformation of the measuring device may itself distort the forces being measured. Methods of measuring force by pressuresensitive discs attached to the foot have also been used, for example by Schwartz and Heath (1949) and Bauman and Brand (1963), but these devices may themselves alter the pattern of walking.

The advent of the force plate allowed Cunningham (1950) to make an accurate measurement of the forces produced in free walking on a rigid surface. These forces could be correlated with the footprint for the whole step, but not with the foot contact area at different phases of the step.

The investigations to be described utilised a force plate modified for simultaneous filming of the contact area of the foot. The recording of information from the force plate was simplified and speeded by a data acquisition system.

\section{MATERIAL AND METHODS}

The force plate is basically a rigid platform on which the subject can walk. The reaction of the foot on the plate is measured by strain gauges arranged to produce outputs dependent on the forces and moments in three mutually perpendicular axes on the surface of the plate. For some purposes the measurement of only the major component, the vertical force, is sufficient. Such a system was developed by Skorecki (1966), in association with Charnley, to study the effect of hip joint replacement on gait.

Where continuous recording of all six forces is required the force plates are of two types, depending on whether the plate is supported by columns or suspended on strips. The column-supported type originated by Cunningham and Brown (1952) has the disadvantage of requiring four substantial supporting tubes; its sensitivity is consequently limited compared with the strip-suspended plate used in the present investigation. This plate, which has been fully described by McLeish and Arnold (1972), is supported and restrained by four vertical and three horizontal spring steel strips to which strain gauges are bonded. Its vertical sensitivity is approximately five times that of the column-supported type. The original force plate was modified for the present studies by cutting a window $27.3 \times 17.5$ centimetres in the steel grid sub-platform and by replacing its wooden surface with a slab of glass 2.5 centimetres thick. Grid lines were marked on the glass as reference axes. Beneath the platform a mirror was mounted at 45 degrees to the vertical, permitting horizontal viewing of the underside of the foot by a cine camera (Fig. 1).

The force plate was mounted flush in the centre of a walkway allowing several steps on either side (Fig. 2). Two micro-switches incorporated in the walkway signalled contact of the foot not being measured. This allowed measurement of progression in steps per minute and defined the time during which both feet were in contact with the ground. The glass plate was illuminated through its rear edge by a 1-kilowatt quartz-iodine strip lamp and the plate was lightly oiled. This oiling of the glass plate produced no sensation of instability in the subjects and did not appear to affect their gait. With this arrangement excellent delineation of the foot contact area was achieved (Fig. 3) and was filmed through the mirror by a cine camera running at 64 frames per second.

The readings from the force plate were fed into a conventional ultra-violet recorder, giving a visual picture of the activity in the strain gauge bridge circuits. However, calculation of results from the ultra-violet recorder traces is tedious

Michael Grundy, F.R.C.S.Ed., Consultant Orthopaedic Surgeon, Blackburn Royal Infirmary, Blackburn, Lancashire BB2 3LR, England. P. A. Tosh, M.Sc., Demonstrator in Mechanical Engineering, University of Manchester Institute of Science and Technology, Manchester, England.

R. D. McLeish, B.Sc., Senior Lecturer in Mechanical Engineering, University of Manchester Institute of Science and Technology, Manchester, England.

L. Smidt, F.Ch.S., S.R.Ch., Lecturer at the Northern College of Chiropody, Salford College of Technology, Salford, England. 
and time-consuming. This was overcome by the simultaneous use of a PDP8/E computer (Digital Equipment Corporation) incorporating a seven-channel analogue-digital convertor. The machine was programmed to sample and store information from all the strain gauges at specified regular intervals and then to produce a punched tape of the stored information.

The tape was later fed into a PDP 15/40 computer programmed to calculate and plot curves of centres of pressure paces so that the foot being studied struck the force plate towards its centre. With a little practice the steps could be started at a point from which the foot consistently struck the force plate without hesitation. The size of the window in the plate was only just big enough to accommodate the foot, but rather than trying to achieve precise steps, the subject was encouraged to walk normally and tests in which the foot did not centre on the plate were discarded.

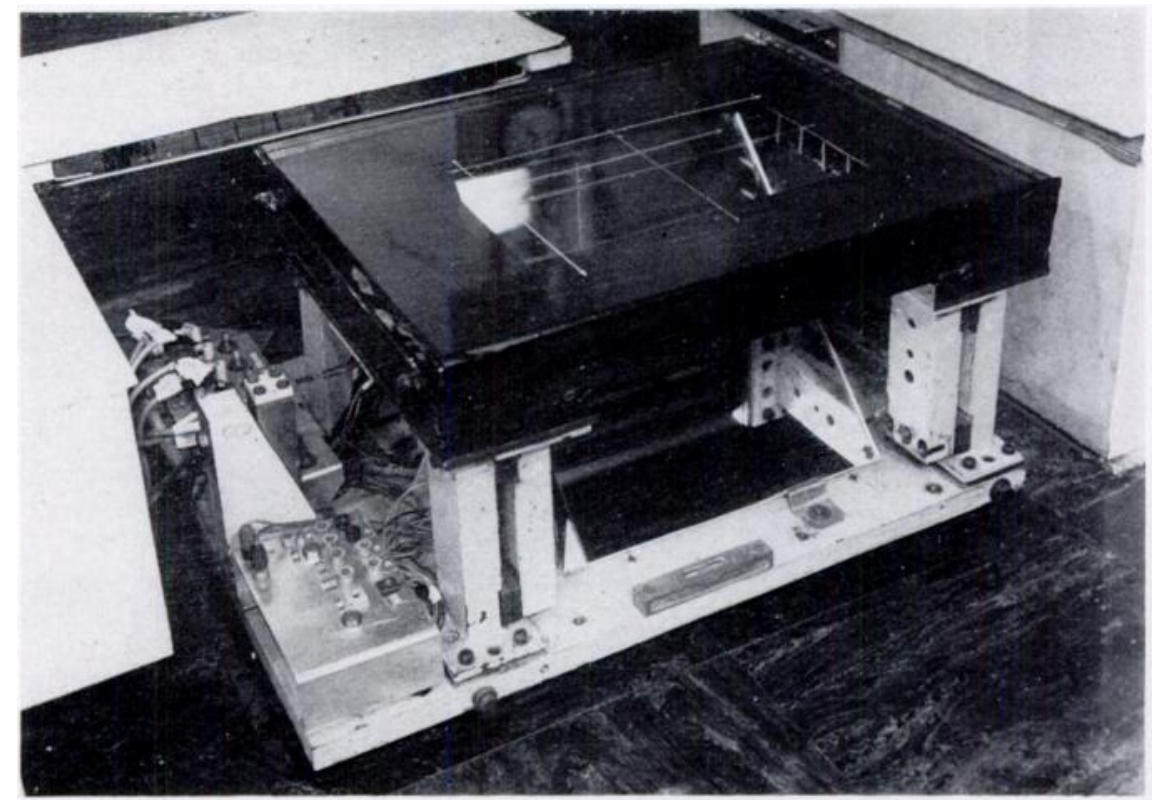

FIG. 1

The modified force plate.

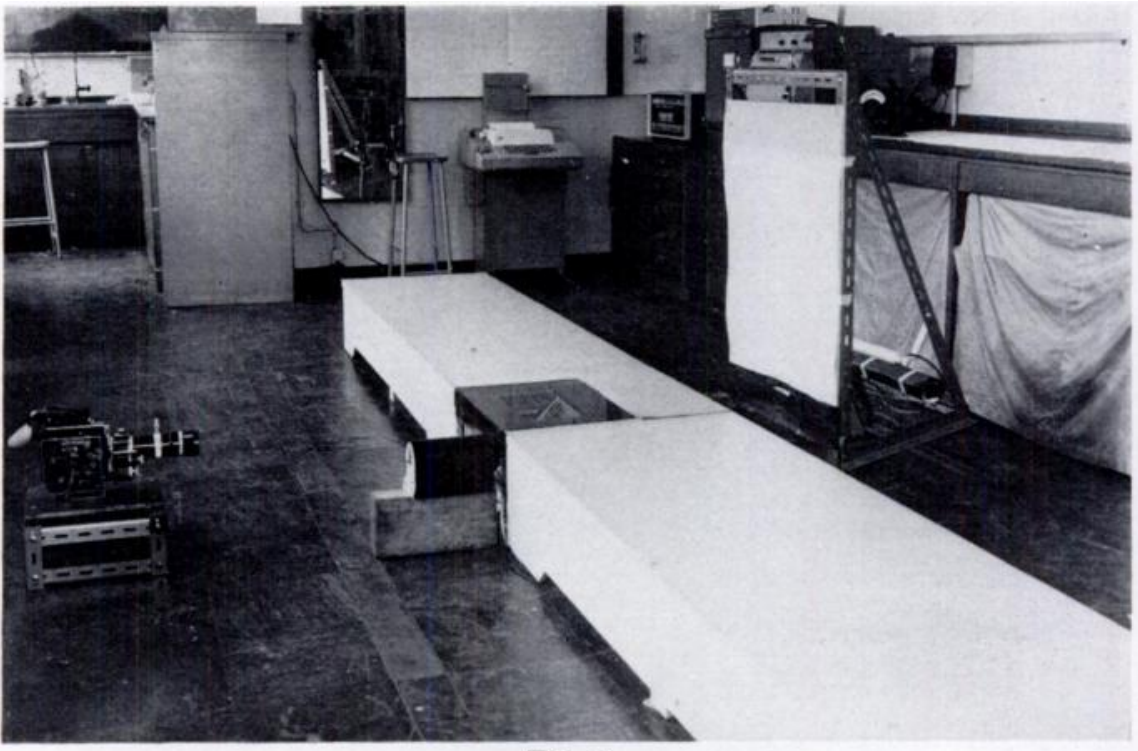

FIG. 2

The experimental arrangement, showing the force plate incorporated in the walkway, with synchronising clock, cine camera and computer.

and the resultant forces. Derived functions such as the velocity of the centre of pressure were also computed. These results were then correlated with the cine film of the foot contact areas by means of a synchronising clock which appeared on the film and which also produced a signal recorded by the computer.

In use a subject was asked to walk up and down the walkway at a normal pace and was helped to arrange his

\section{RESULTS}

Tests were made on a total of sixteen subjects with normal or abnormal feet, both barefoot and shod. The tests were frequently duplicated, in some cases after an interval of several weeks. The results for a given subject were consistent and repeatable. 
There were ten adult male subjects with normal feet. "Normal" in this context does not exclude minor degrees of postural flat foot. A typical result is illustrated in Figure 4 and will be described in detail.

The upper trace represents the vertical force under the left foot as the subject walked from right to left. Force is measured on the upper vertical axis, and distance travelled along the horizontal axis. Arrows $\mathrm{T}$ and $\mathrm{H}$ are the times of toe-off and heel-strike of the opposing foot for the preceding and following steps. The interval between $\mathrm{T}$ and $\mathrm{H}$ is thus the time during which only

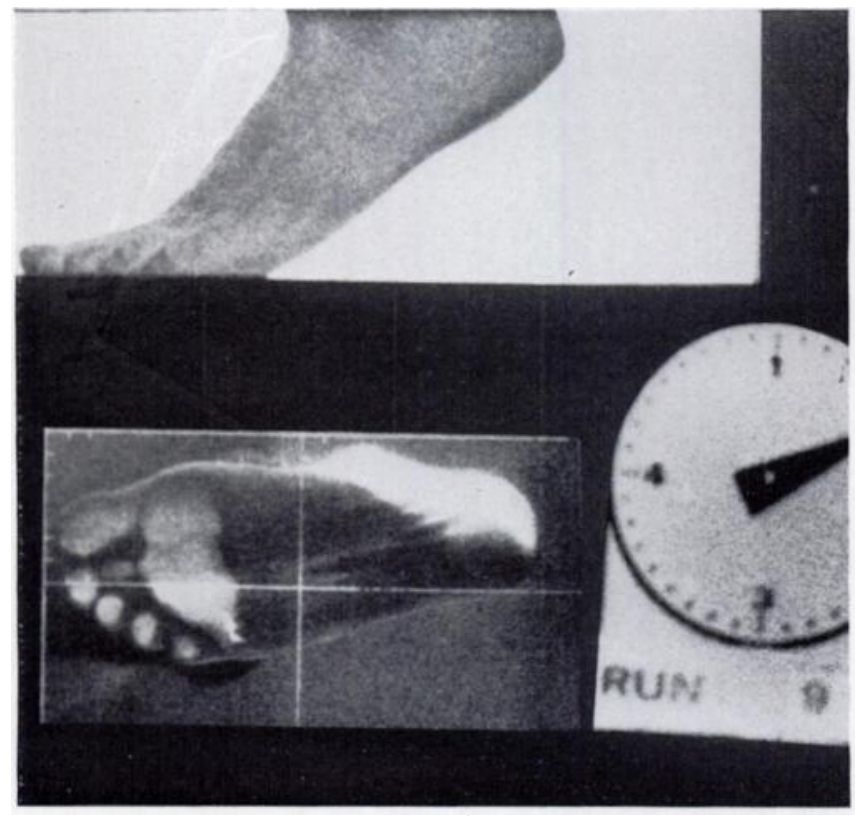

Fig. 3

A frame from the cine film showing delineation of the foot contact area.

the left foot is in contact with the ground. The points on the curve are at equal times of one twenty-fifth second.

The middle curve is on the same axes as the upper curve and represents horizontal forces in the line of progression. The force is forward at heel contact and backward at toe-off.

The lower trace is the path followed by the centre of pressure of the foot over the surface of the force plate and relative to the whole footprint, which is superimposed.

The magnitude and relative proportions of the vertical and horizontal forces correspond to those described in previous investigations by Cunningham (1950) and by Bresler and Frankel (1950), but the general shape of the curves does not. The reason for this is that force is displayed against distance instead of time. Instead of a symmetrical twin-peaked curve, an asymmetrical curve is produced which shows very clearly the following features of normal gait.

1) Immediately after heel-strike there is a rapid build-up of vertical force and progression forward of the centre of pressure (the crosses marking equal time intervals are far apart). However, the force does not reach a maximum while the heel alone is in contact with the ground. The first peak is well forward of the ball of the heel, and cine films show that the lateral border of the heel and the metatarsal heads have both made contact with the plate at this stage. 2) During the next phase of gait, while the heel is still in contact with the plate, the centre of pressure continues to move forwards rapidly as force on the heel decreases and force on the metatarsal heads increases. The line followed by the centre of pressure is the midline of the foot, well away from the contact area of the lateral border of the foot. This suggests that weight is being moved directly from heel to forefoot and that the midfoot plays little part in transferring the load. 3) When the vertical force, which has dropped slightly in the midfoot phase, begins to rise to its second peak the heel is leaving the ground and the toes are making contact. This period, when the whole load is taken on the forefoot, is long relative to the period of heel contact, as can be seen by the close clustering of time-markers. This clustering occurs under the metatarsal heads. The magnitude and duration of force in this area suggests that the forefoot is much more important than the hindfoot in transferring force. Further calculations which were made to substantiate this will be described below. 4) During the forefoot phase the centre of pressure remains in the region of the metatarsal heads even after the toes have made full contact with the plate. This implies that the toes transmit very little force. Further confirmation is obtained in the period after the metatarsal heads have left the ground and the toes alone are in contact: the vertical and horizontal forces drop off very rapidly indeed. The line of the centre of pressure suggests that the small force exerted by the toes is transmitted chiefly through the first and second toes.

As mentioned above we were impressed by the apparently high force-transmitting function of the forefoot compared with the hindfoot and have attempted to express this quantitatively. The simplest measurement is the length of time in which the metatarsal heads are transmitting most of the force, generally related to the easily calculated mean velocity of the centre of pressure at the metatarsal heads. The lower this velocity, the greater is the time during which force is transmitted. In eight of the ten normal subjects this was relatively low, from six to ten centimetres/second (mean 7.86). This can be used as a basis for comparison with abnormal subjects.

The second index was the ratio between the forefoot and heel impulses. This was obtained by calculating and comparing the proportions of the total vertical impulse at two points. These points were at the interval between the first and second metatarsal heads and at the centre of the heel. "Impulse" in this sense refers to the integral of force against time, or the area under the force time curve. This ratio was called the index of forefoot function and in eight of ten normal subjects had a value ranging from 2.02 to 5.05 (mean 3.33). In general this means that the load-bearing function of the forefoot is three times that of the hindfoot.

There were two normal subjects in whom the results did not fall within the same range as the remaining eight; they showed a more rapid transference of pressure over the forefoot, with consequent higher centre of pressure velocities and lower indices of forefoot function. These probably represent a variation of the normal pattern and overlap the less extreme results obtained in abnormal 
feet and described below. The summarised results for normal subjects are shown in Table I.

TABLE I

The Findings in Normal Subjects Walking Barefoot

\begin{tabular}{rcc}
\hline Subject & $\begin{array}{c}\text { Mean velocity of the centre } \\
\text { of pressure under the } \\
\text { metatarsal heads }(\mathrm{cm} / \mathrm{sec})\end{array}$ & $\begin{array}{c}\text { Index of forefoot function } \\
\text { (ratio of forefot impulse } \\
\text { to heel impulse) }\end{array}$ \\
\cline { 2 - 3 } 1 & 10.0 & 3.17 \\
2 & 6.0 & 3.02 \\
3 & 6.0 & 2.24 \\
4 & 6.0 & 2.02 \\
5 & 10.0 & 3.05 \\
6 & 6.0 & 4.99 \\
7 & 7.0 & 3.58 \\
8 & 6.0 & 3.08 \\
9 & 19.0 & 1.72 \\
10 & 22.0 & 1.58 \\
\hline
\end{tabular}

A further set of recordings was made of one of the normal subjects wearing a variety of footwear (Table II). The effect of increasing the rigidity of the sole of the shoe is apparent. Figure 5 illustrates the type of recording

TABLE II

The Findings in a Normal Subject Walking Shod

\begin{tabular}{|c|c|c|}
\hline Type of footwear & $\begin{array}{l}\text { Mean velocity of the } \\
\text { centre of pressure } \\
\text { under the metatarsal } \\
\text { heads }(\mathrm{cm} / \mathrm{sec})\end{array}$ & $\begin{array}{l}\text { Index of forefoot } \\
\text { function (ratio of } \\
\text { forefoot impulse } \\
\text { to heel impulse) }\end{array}$ \\
\hline Barefoot & $9 \cdot 0$ & $3 \cdot 27$ \\
\hline \multicolumn{3}{|l|}{$\begin{array}{l}\text { Footwear in order of } \\
\text { increasing rigidity of sole }\end{array}$} \\
\hline $\begin{array}{l}\text { Plimsoll } \\
\text { Leather soled shoe } \\
\text { Hiking boot } \\
\text { Wooden clog }\end{array}$ & $\begin{array}{r}9 \cdot 0 \\
14 \cdot 0 \\
17 \cdot 0 \\
20 \cdot 0\end{array}$ & $\begin{array}{l}2 \cdot 38 \\
2 \cdot 37 \\
2 \cdot 39 \\
2 \cdot 21\end{array}$ \\
\hline \multicolumn{3}{|l|}{ Modified footwear } \\
\hline $\begin{array}{l}\text { Metatarsal bar. } \\
\text { Heel raise } 3 \mathrm{~cm} . \\
\text { Medial heel raise } 6 \mathrm{~mm}\end{array}$ & $\begin{array}{l}19 \cdot 0 \\
13 \cdot 0 \\
10 \cdot 0\end{array}$ & $\begin{array}{l}1 \cdot 20 \\
1.90 \\
2 \cdot 18\end{array}$ \\
\hline
\end{tabular}

obtained with a semi-rigid sole and can be compared with the barefoot recording in Figure 4. The peak of pressure under the metatarsal heads becomes spread over a longer distance and the centre of pressure moves more rapidly forwards. Increasing the rigidity causes a progressive increase in the mean velocity of the centre of pressure at the metatarsal heads and a reduction in the index of forefoot function.

A metatarsal bar positioned behind the metatarsal heads had even more effect than a stiff sole in reducing the function of the forefoot. A raised heel also reduced forefoot function, though to a lesser extent. The wearing of a medial heel raise produced no significant deviation laterally of the centre of pressure, but all the shoes tended to constrain the centre of pressure into following the midline of the shoe rather than allowing the medial

TABLE III

The Findings in Abnormal Subjects

\begin{tabular}{lllll}
\hline Subject & Condition & Footwear & $\begin{array}{l}\text { Mean velocity } \\
\text { of the centre } \\
\text { of pressure } \\
\text { under the } \\
\text { metatarsal } \\
\text { heads }(\mathrm{cm} / \mathrm{sec})\end{array}$ & $\begin{array}{l}\text { Index of fore- } \\
\text { foot function } \\
\text { (ratio of fore- } \\
\text { foot impulse to } \\
\text { heel impulse) }\end{array}$ \\
\hline 11 & Hallux rigidus & Barefoot & $10 \cdot 0$ & 2.36 \\
12 & Varus heel & Barefoot & 30.0 & 0.23 \\
13 \\
14 \\
15
\end{tabular}

deviation towards the first and second metatarsal heads and toes seen in barefoot walking.

Six subjects with abnormal feet were all recorded walking barefoot, and two were recorded wearing surgical shoes. One subject had a hallux rigidus of moderate degree and one had a varus deformity of the heel with painful callosities on the lateral border of the foot. The remaining four subjects all had gross hallux valgus with deformities of the lesser toes, metatarsal callosities and complaints of metatarsalgia. The summarised test results are shown in Table III.

Figure 6 shows a representative record of a subject with metatarsalgia. Following the initial build-up of vertical force, the centre of pressure moves slowly forward in the region immediately in front of the heel. Although both heel and metatarsal heads are in contact with the ground at this time, most of the weight remains on the heel instead of being transferred forward. As the body moves forward the heel is eventually raised from the ground; the centre of pressure then accelerates forward until the whole foot has left the ground after only a short period of support by the metatarsal heads. The magnitude, as well as the duration, of force under the metatarsal heads is reduced, there being no second peak on the curve. The toes do not function. In this subject the velocity of the centre of pressure under the metatarsal heads was a rapid 25.0 centimetres/second and the index of forefoot function was reduced to $0 \cdot 81$. All four subjects with metatarsalgia had similar recordings to the one illustrated, and the wearing of surgical shoes in two had a variable effect in increasing forefoot function. 
The subject with a varus heel produced a significant lateral deviation of the line of the centre of pressure and had a low index of forefoot function. The subject with a hallux rigidus had an index of forefoot function at the lower end of the normal range. computer analysis and plotting of results, has enabled detailed investigation of the centres of pressure beneath the foot.

In normal barefoot walking a characteristic pattern emerges. The forces transferred by the heel and meta-

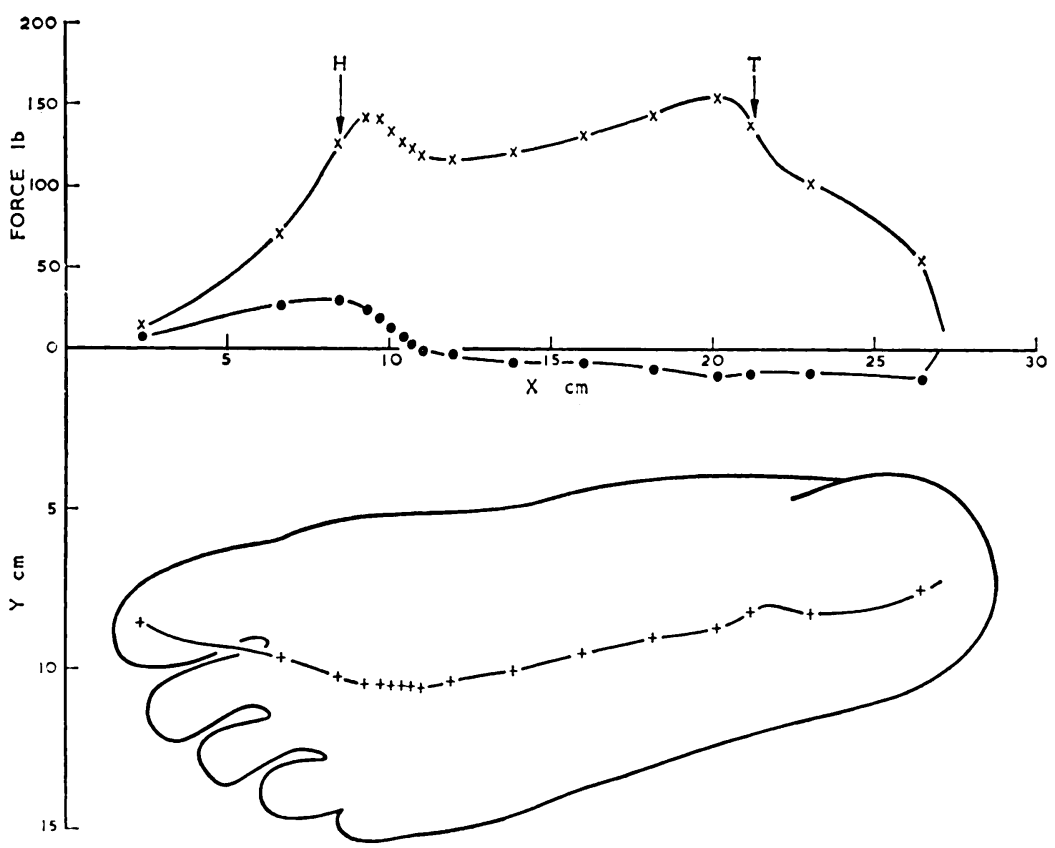

Fig. 4

The forces and centres of pressure recorded in a normal subject walking barefoot.

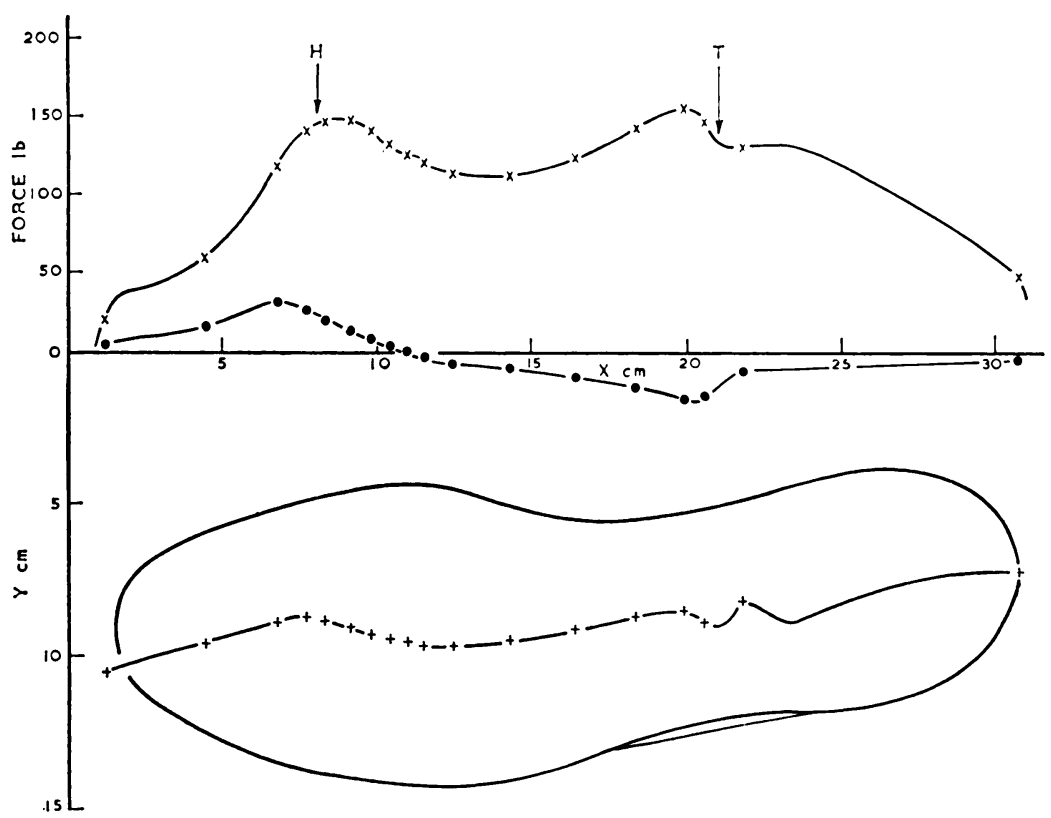

Fig. 5

The forces and centres of pressure recorded in a normal subject wearing a shoe.

\section{CONCLUSIONS}

The experimental arrangement described in this paper, combining cine films, a force plate of high accuracy and tarsal heads are high compared with the low forces transferred from midfoot and toes. This is in accord with findings recently reported by Stott, Hutton and Stokes (1973). Of greater importance is the proportion of 
the total impulse transferred by the forefoot. Although the forefoot sustains only slightly greater loads than the heel, it does so for a much longer period in the walking cycle. Our results suggest that the load-bearing function of the forefoot is about three times that of the heel.

When shoes are worn the load-bearing function of the forefoot is progressively reduced as the rigidity of the sole increases. This has important implications in the design and construction of footwear if it is to allow the foot to function normally. The effects of different types of footwear, including high heels, are presently being investigated.

In the subjects with metatarsalgia the function of the forefoot was greatly reduced, the heel taking a greater part of the total impulse. This highlights the importance of the forefoot and the extent to which its function can be inhibited by pain or deformity. It is hoped that the method described may be used to compare the therapeutic effects of different methods of treatment of foot disorders.

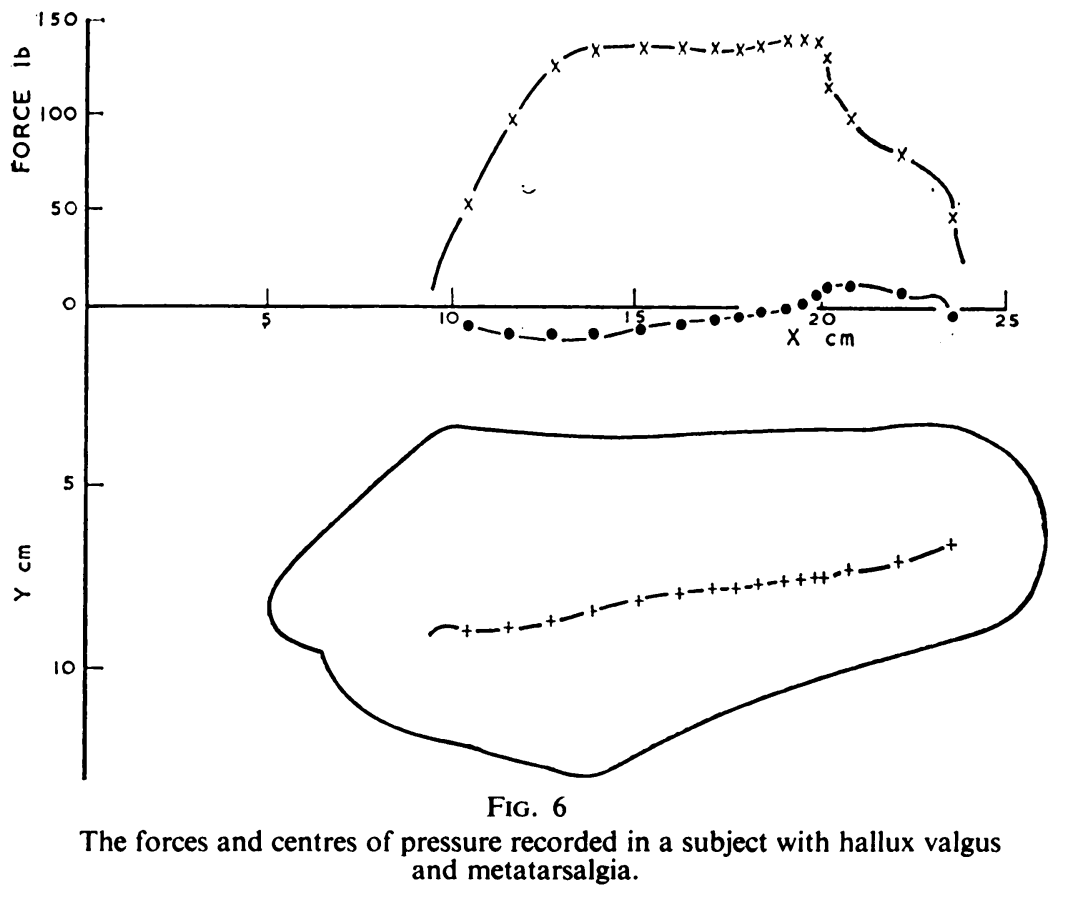

Acknowledgements are due to the Science Research Council for a grant to Mr P. A. Tosh, and also to Mr John Howe for the photographic work.

\section{REFERENCES}

Barnett, C. H. (1954) A plastic pedograph. Lancet, 2, 273.

Bauman, J. H., and Brand, P. W. (1963) Measurement of pressure between foot and shoe. Lancet, 1, 629-632.

Bresler, B., and Frankel, J. P. (1950) Forces and moments in leg during level walking. Transactions of the American Society of Mechanical Engineers, 72, 27-36.

Cunningham, D. M. (1950) Components of floor reactions during walking. Prosthetic Devices Research Project, Institute of Engineering Research, University of California, Berkeley. National Research Council, Advisory Committee on Artificial Limbs. Series 3, Issue 14.

Cunningham, D. M., and Brown, G. W. (1952) Two devices for measuring the forces acting on the human body during walking. Proceedings of the Society for Experimental Stress Analysis, 9, part 2, 75-90.

Elftman, H. (1934) A cinematic study of the distribution of pressure in the human foot. Anatomical Record, 59, 481-491.

McLeish, R. D., and Arnold, D. A. (1972) A foot-ground reaction force plate. Joint British Committee for Stress Analysis. Proceedings of Conference on the Recording and Interpretation of Engineering Measurements, pp. 75-80. London: Institute of Marine Engineers.

Schwartz, R. P., and Heath, A. L. (1949) The oscillographic recording and quantitative definition of functional disabilities of human locomotion. Archives of Physical Medicine, 30, 568-578.

Skorecki, J. (1966) The design and construction of a new apparatus for measuring the vertical forces exerted in walking: a gait machine. Journal of Strain Analysis, 1, 429-438.

Stott, J. R. R., Hutton, W. C., and Stokes, I. A. F. (1973) Forces under the foot. Journal of Bone and Joint Surgery, 55-B, 335-344. 